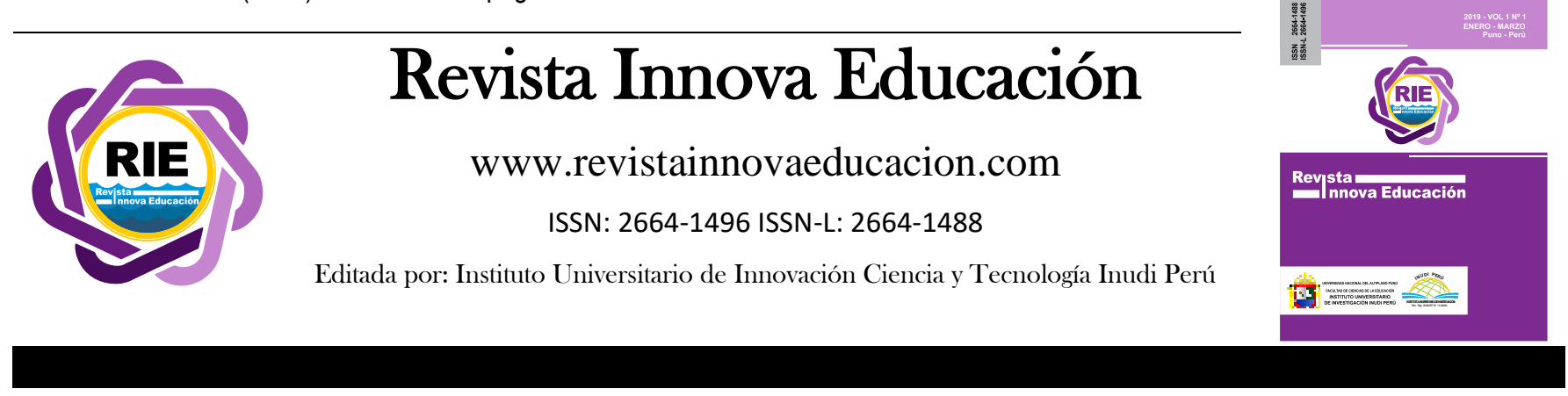

\title{
Política social educativa y violencia escolar: brechas en su atención
}

\author{
Educational social policy and school violence: gaps in their attention \\ Caridad Cala-Montoya ${ }^{1}$ \\ Universidad de Oriente, Santiago de Cuba - Prov. de Oriente, Cuba \\ https://orcid.org/0000-0002-0801-6467
}

DOI: https://doi.org/10.35622/j.rie.2020.01.003

Recibido 20/12/2019/ Aceptado 24/01/2020 Publicado 29/01/2020

\begin{tabular}{ll}
\hline ARTÍCULO ORIGINAL & $\begin{array}{l}\text { El presente trabajo brinda un análisis crítico a la política social educativa cubana en el } \\
\text { PALABRAS CLAVE }\end{array}$ \\
& $\begin{array}{l}\text { tratamiento a la violencia escolar, en aras de contribuir a su perfeccionamiento y a } \\
\text { visibilizar dicha problemática. La investigación realizada asume como estrategia } \\
\text { metodológica la triangulación de metodologías, y tiene como unidad de análisis } 4\end{array}$ \\
& centros escolares de la enseñanza secundaria del municipio Santiago de Cuba. Del \\
& análisis de los resultados se destaca que, si bien el país se encuentra en un franco \\
Fisuras educativas, & recorrido hacia la visibilidad de este flagelo, la omisión del término en los decretos y \\
política educativa, & resoluciones ministeriales de la política educativa favorece al desconocimiento que \\
violencia escolar. & sobre la misma persiste. En ella, los acercamientos tangenciales al tema se circunscriben \\
& a conductas antisociales del estudiantado y a sus contextos de vulnerabilidad; no \\
& teniendo en cuenta ni a profesores, familiares o demás actores escolares que participan \\
& en su construcción. Igualmente, en la elaboración de estrategias para su atención, se \\
& tiende a preponderar la participación de grupos minoristas distantes de la realidad \\
& escolar, y proliferan fisuras en las directrices de capacitación docente que se \\
implementan.
\end{tabular}

KEYWORDS

Fisuras educativas, política educativa, violencia escolar.

\begin{abstract}
This paper provides a critical analysis of Cuban educational social policy in the treatment of school violence, in order to contribute to its improvement and to make visible this problem. The research carried out takes as a methodological strategy the triangulation methodological, and assumes the basic secondary schools of the Santiago de Cuba municipality as empirical references for their development. From the analysis of the results it is highlighted that, although the country is on a clear path towards the visibility of this scourge, the omission of the term in the ministerial decrees and resolutions of the educational policy favors its ignorance. In it, the tangential approaches to the subject are limited to students' antisocial behaviors and their contexts of vulnerability; not considering teachers, family members or other school actors involved in its construction. Likewise, in the development of strategies for their attention, the participation of retail groups distant from school reality tends to prevail, and cracks proliferate in the teacher training guidelines that are implemented.
\end{abstract}

${ }^{1}$ Correspondencia: carla87@uo.edu.cu 


\section{INTRODUCCIÓN}

Uno de los pilares de la sociedad cubana, a partir del triunfo revolucionario, ha sido velar por el pleno desarrollo de los cubanos, vinculado a las realidades contextuales de cada época; con énfasis en el cuidado y protección de los niños, adolescentes y jóvenes. En dicho proceso, tanto el sistema educativo como la política social educativa ocupan un lugar primordial. Sus primeros aciertos, en aras de surcar las barreras de la desigualdad de acceso y discriminación de los escolares afrodecendientes y de sectores empobrecidos del período previo a 1959 se evidenciaron con la inserción masiva en las aulas, sin distinción de raza o credo y desde el fomento de la igualdad en educadores y colegiales (Constitución de la República, 1976; González y Reyes, 2010; Chávez y Deler, 2013; Labacena, 2015). Además de la eliminación de las escuelas privadas, incremento de los centros escolares y docentes, para acoger a la masa estudiantil que emergía, así como para la preparación de los profesores (García, 1998; Fabregat, 2016). No obstante, pervivía del viejo modelo educativo derivado de sistema educativo español, la aplicación de castigos físicos y otros procedimientos violentos a los estudiantes en algunos colegios, con tipologías correctivas muy similares derivadas del siglo XIX y presentes durante el XX (Cordoví, 2009, 2012). Igualmente, vestigios racistas y clasistas se presentaban en el escenario escolar y favorecían al trato desigual y excluyente hacia los estudiantes (Zabala, 2008; Cala, 2019).

En aras de hacer frente a estas realidades subyacentes, enmarcadas en los estudios de la disciplina escolar (Cordoví, 2009, 2012) y elevar la calidad de nuestro sistema de enseñanza, el país ha transitado por varias etapas de perfeccionamiento de su estructura organizacional y directrices políticas (Ministerio de Educación, 2015). Súmese, también, la creación de instituciones (centros de diagnóstico y de atención a menores), la firma de acuerdos políticos internacionales y normativas educativas válidos para la atención a los colegiales, así como a los comportamientos anómicos (violentos) que laceran las relaciones escolares. Disponiéndose hacia el sector educativo, el 13\% del producto interno bruto del país, según informe del Banco Mundial (Borroto, 2016, p.78). Indistintamente, en los últimos años y a tenor de los cambios socioeconómicos, políticos, demográficos y culturales por los que peregrina las sociedad cubana, comienzan a despuntar de forma explícita estudios de violencia que dan cuenta de brechas significativas en las pautas de dicha política para su tratamiento (Regueira, 2008; Rodney, 2010a y b; Santiesteban, 2013; Rodney, y García, 2014; Rodney, Lorenzo, Cruz y Muñoz, 2015; 
Sampedro, 2017; Campañat, 2017; Cala y Caraballo, 2017; Rodríguez y Ramos, 2018; Cala y Benítez, 2018; Torres et al., 2019; Benítez, 2019, y otras²).

Se alude de esta forma a la irrupción de discriminación, agresión física, verbal, psicológica y acoso entre estudiantes y de profesores a ellos como prácticas culturales naturalizadas en la dinámica escolar; sin embargo, aún se encuentra el país en un cierto velo hacia su reconocimiento. La omisión que la acompaña, como expresión de violencia escolar, puede asociarse a los altos niveles de escolarización (100\%), promoción escolar (92,5\%) (Oficina Nacional de Estadística e Información, 2019, p.62) y bajos de repitencia de los que internacionalmente goza el país; además del débil tratamiento a la temática y a su construcción dentro de la política educativa.

En tal sentido, la presente investigación tiene como finalidad realizar un análisis crítico de la política social en el ámbito educativo respecto al tratamiento que tiene la violencia escolar; tomando como referente escuelas del municipio Santiago de Cuba. Con este, se pretende no solo visibilizar la problemática en el territorio santiaguero y alertar de sus consecuencias imprevistas para las relaciones escolares y el sistema educativo, sino potenciar un conjunto de acciones que tributen a perfilar las directrices de la política educativa sobre dicho particular.

Cabe destacar que, al hablar de política social nos referimos a esta tipología de política pública-decisiones, acciones e inacciones de las autoridades públicas para resolver o prevenir una situación definida como problemática (Velásquez, 2009, p. 156)-, encargada de velar por la calidad de vida de los seres humanos "mediante la materialización de sus derechos sociales, económicos, culturales y otros, a través de la redistribución, la inclusión social y la generación de oportunidades" (Vargas, 1999, p. 6 en Velásquez, 2009, p. 186).

En el marco del ámbito educativo, estas se relacionan con el desarrollo social de nuestro país que, junto a otras naciones latinoamericanas, hoy enfrentan retos sustanciales para el “crecimiento económico y la equidad social” (Borroto, 2016, p. 77). La complejidad estructural de donde resulta, en nuestro escenario, implica sortear los constantes embates del bloqueo económico y las desigualdades acrecentadas en la nación (Espina, 2014; Domínguez, 2016, Zabala et al, 2018), en interconexión con el incremento del trabajo por cuenta propia, las altas tasas de migraciones y de envejecimiento poblacional, y la crisis financiera de los últimos tiempos

\footnotetext{
${ }^{2}$ Otras investigaciones, además de las expuestas, se ubican en La Habana, Las Tunas, Villa Clara, Santiago de Cuba y Guantánamo. Entre ellas: Romero, 2008; Aguilera et al., 2011; González y Aramayo, 2011; Santiesteban, 2013; Campañat, 2013; Lambert, 2013; Balcindes, 2014; Rodney, Rodríguez y del Valle, 2015; Rodríguez, Rodríguez. y Ledón, 2015; Garcés, 2015; Díaz, Santiesteban y Matos, 2015, 2016; Isalguí 2015; Rosabal, 2015; Leyva, 2015; Guevara, Ferrer, Norman, Suz, 2016; Cala, et al, 2016; Hernández, 2016; Dexie, 2016, Servicios de Noticias de la Mujer de Latinoamérica y el Caribe, 2016; Manreza, Barriel, y González, 2017; Lotti, 2017; Más, 2018; Hernández, 2018; Suárez et al., 2018; Cala et al 2018a y b; Cala, 2019; Arlet, 2019.
} 
(Naranjo, Figueroa y Cañizares, 2015; Labacena y Tamayo, 2018). Aun así, su eje de acción se enmarca en impulsar el desarrollo integral y pleno de los seres humanos, bajo los principios de: gratuidad, obligatoriedad de la enseñanza primaria y secundaria, vínculo del trabajo con el estudio, fomento de la investigación y la relación teoría- práctica (Chávez y Deler, 2013) quienes en conjunto tributan a la formación integral de los colegiales.

En la investigación, la violencia escolar se asume como esa "realidad cualitativamente nueva resultante de las relaciones recíprocas entre actores, individuales y/o colectivos en el marco escolar, que emerge en formas de daño físico, verbal, psicológico o económico de uno contra el otro o contra ambos a la vez, autoorganizado por medio de unos vehículos comunicativos que orientan el accionar de los sujetos durante sus intercambios" (Cala, 2019, p. 20). A tenor del estudio, aunque se tome como base la matriz relacional de la irrupción de la violencia escolar, a partir de las relaciones recíprocas entre los actores y entre estos y las distintas estructuras y sistemas por las que atraviesan, se reconoce que, en la construcción de dicha violencia, desde el sistema normativo de la política social educativa, confluyen integradamente otros sistemas como: el familiar, el simbólico-comunicativo, el del poder, el cultural, comunitario, etcétera.

\section{MÉTODO}

La investigación responde a un estudio de naturaleza prospectiva y descriptiva que tuvo como estrategia metodológica la triangulación (Denzin, 1970). La misma se apoyó en la triangulación de metodologías -cualitativa y cuantitativa- (Denzin, 1970, p. 301, cursiva añadida) para el análisis crítico de la política social en el ámbito educativo respecto al tratamiento a la violencia escolar. El estudio toma como unidad de análisis 4 escuelas de la enseñanza secundaria del municipio Santiago de Cuba.

En aras de profundizar en cómo existen vacíos y fisuras en la política educativa que tributan, directa e indirectamente, a la emergencia de la violencia escolar, sin distorsionar ni someter a experimentos la cotidianeidad educativa desde donde se aprecian, se emplean entre las técnicas de la metodología cualitativa: la observación directa (participante), la entrevista en profundidad y el análisis de documentos. Para ello, se selecciona un "muestro intencional opinático y teórico-" (Ruiz, 1996, p. 95).

En lo concerniente a la metodología cuantitativa, el muestro fue probabilístico y aleatorio simple (Ruiz, 1999; Hernández, 2014) teniendo como técnica el cuestionario a estudiantes, familiares y profesores. Este permite, desde las percepciones de los sujetos escolares y la realidad educativa colectivamente construida en su interacción, identificar, a partir de una visión más 
representativa, las diversas formas de emergencia de la violencia en el centro, en interconexión con los lineamientos y proyecciones de la política educativa.

El cuestionario a estudiantes y familiares respondió a un muestreo estratificado por los grados escolares: 7mo., 8vo. y 9no.; mientras, en los docentes se asoció a su población por escuelas. Ambos cálculos tuvieron un margen de error muestral de $10 \%$ y un nivel de confianza del 95,57. La probabilidad de que todos pudieran ser escogidos por constituir elementos de la muestra se garantizó a través del muestreo aleatorio simple (al azar). En total se encuestaron 425 estudiantes, 151 docentes y 460 familiares.

\section{RESULTADO Y DISCUSIÓN}

\section{Los caminos silenciados entre la política educativa y la violencia escolar.}

En la materialización del cuidado y protección del menor en el ámbito escolar, diversas son las normativas, resoluciones y decretos creados como garantes de su educación sexual y formación integral. No obstante, al interior del trabajo preventivo y la matriz profilaxis que de ellas debe derivar, persiste una carencia en la atención a la violencia escolar. Expreso en la inexistencia del término en sus artículos e incisos.

En tal sentido, los resultados del análisis de documento permiten aseverar que existen, desde las normativas, resoluciones y decretos educativos acercamientos tangenciales a la violencia escolar. Ello se reafirma, toda vez que se tiende a minimizar su magnitud y significación social al subsumirla dentro de categorías cautelosas y sutiles de agravio, como: indisciplinas graves, conductas antisociales y hechos antisociales de elevada peligrosidad social (Artículo 2, Decreto Ley 64/1982), discriminación por cuestiones de género (Resolución Ministerial 139/2011) y conductas muy graves, graves y menos graves entre y de los escolares (Resolución Ministerial 11/2012). Fisura que se extrapola hacia las proyecciones de la política social educativa, hasta el 2021, donde su presencia debe inferirse bajo la dimensión del perfeccionamiento del sistema de enseñanza, en función de la elevación de la calidad y rigor del proceso docente educativo (Lineamientos de la Política Económica y Social del Partido y la Revolución para el período 20162021, p.22); más, al no contar con indicadores que aludan a este flagelo, su atención pasa inadvertida desde las relaciones escolares.

De igual forma, desde su matriz direccional de concepción y emergencia dichas prácticas antisociales (cual expresión de violencia), limitan su spectrum interactivo a las relaciones entre estudiantes y escasamente de profesores a estos. Realidad que deja fuera al resto de los actores individuales y colectivos que participan en su construcción y reproducen las miradas 
internacionales sobre dicho particular, donde el bullying toma un lugar primordial (Olweus, 2011; Pérez, 2017; Astor, 2017, Unesco, 2019).

En el análisis del accionar preventivo, a tenor de los instrumentos normativos aludidos, subsiste cierta tendencia al diagnóstico y caracterización de los escenarios de vulnerabilidad que afectan al escolar, pero distantes de asumir el propio centro como un espacio de construcción y reconstrucción de la violencia en su interior. En tal sentido, las miradas a la escuela se remiten al diagnóstico psicosocial del sujeto (estudiante), más no a las estructuras relacionales que se conforman en ellas, y que de estas derivan (Resolución Ministerial 111/2017; Protocolo de violencia, 2017).

Desde el marco de la capacitación del claustro docente, base del lineamiento 120 de la proyección de la política social educativa para el 2021, es válido destacar la voluntad de introducir la violencia escolar en los seminarios metodológicos a nivel nacional, entre 2016-2017 (entrevista a jefa del equipo municipal de atención al trabajo preventivo). Sin embargo, irrumpen brechas operacionales en su implementación en los centros, al igual que vacíos cognoscitivos en su concepción.

Respecto a las brechas operacionales, sus fisuras germinan ante la precaria referencia de indicadores para su control y evaluación a tenor de las estrategias de prevención que dimanan desde cada escuela, para la atención a este flagelo. De ahí que pasen inadvertidas en las dimensiones del trabajo preventivo a nivel municipal y en la evaluación integral del proceso de enseñanza-aprendizaje. Concretamente, ellas, tienden a reducirse a la cuantificación de los colegiales fumadores, los que ingieren bebidas alcohólicas, los procedentes de familias disfuncionales, las embarazadas, etc. Mientras, en la evaluación integral, se enmarcan en el control de las acciones dirigidas hacia la capacidad de dirección, gestión de dirección, funcionamiento del consejo de escuela, y el tratamiento y seguimiento a los estudiantes que incumplen los deberes escolares (Dirección Municipal de Educación, 2019). Tales dimensiones, al no abordar las formas relacionales y de emergencia de la violencia en las escuelas, tributan indirectamente a su legitimación. Además, dichos elementos dan cuenta del desconocimiento profeso en la concepción del fenómeno, al reducir su expresión a la agresión entre iguales $(96,5 \%)$ y de profesores a estudiantes $(67 \%)$-cuestionario a profesores y familiares-.

En este mismo orden de ideas, en el marco de la observación directa o participante (Ruiz, 1996, 26), se percibe, a partir de sus resultados, una cierta tendencia a banalizar la existencia de los reglamentos escolares, pues se incumplen con asiduidad sus normativas rectoras. Asimismo, su resguardo a manos de los directivos de los centros tributa a la pervivencia de su 
desconocimiento por parte de los actores escolares, el cual se hace presente casi siempre cuando irrumpen situaciones violentas que traspasan dichos recintos.

Las secuelas indirectas de lo enunciado tributan, junto otras realidades (violencia intrafamiliar y comunitaria, crisis socioeconómica, etc.), al déficit cognoscitivo de los actores escolares respecto a la violencia en la entidad. Tal es así que, solo el 35\% de estos, entre estudiantes, docentes y familiares (resultados del cuestionario) alega la existencia de la violencia en su centro, pero remiten, cómo: los gritos, las humillaciones, las amenazas y otras prácticas violentas que se reflejan en el gráfico 1, constituyen una cotidianeidad en las escuelas.

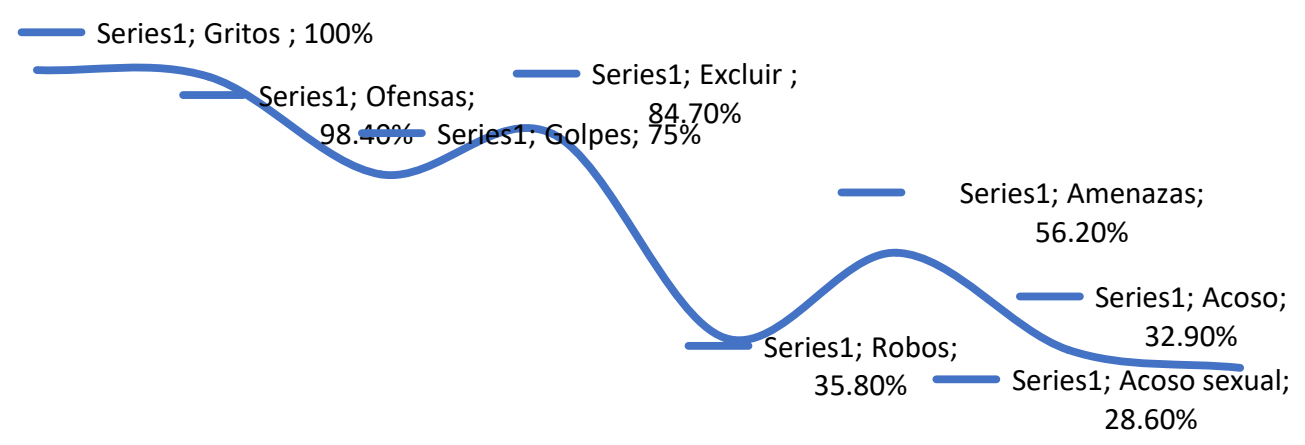

Figura 1: Gráfico1- Formas externalizadas de la violencia escolar. Elaborado por la autora a partir de los datos del cuestionario a estudiantes y profesores.

En esencia los resultados del gráfico expuesto, permiten advertir, cómo los gritos en un 100\%; las ofensas para un 98,4\%; la exclusión en las interacciones escolares con un 84,70\%; los golpes en un $75 \%$ y las amenazas con un 56,20\% laceran con mayor impacto la dinámica educativa, externalizando formas de violencia en el contexto escolar. Asimismo, aunque otras afecciones como el robo con un $35,8 \%$, el acoso para un $32,90 \%$, y el acoso sexual para un $28,6 \%$ no poseen cifras tan elevadas, sí posibilitan ilustrar nuevas formas de irrupción de este flagelo en el contexto santiaguero.

Al margen del carácter intencional o no que tengan, la presencia reiterada de estas prácticas en las escuelas, según datos del cuestionario a estudiantes, se configura principalmente en las relaciones entre escolares $(84,70 \%)$, de profesores a estudiantes $(76,9 \%)$, de estos hacia los docentes $(57,40 \%)$ y de familiares a docentes $(47,9 \%)$ en aulas, pasillos y baños. En su mayoría, las formas externalizadas de la violencia escolar apuntadas, son recibidas con sonrojos o cual forma de agravio entre unos y otros durante sus interacciones. Realidad que, a su vez, da cuenta 
del distanciamiento entre los estudios del tema en el país y las propias vertientes políticas que lo sustentan. En otras palabras, aludimos a cómo cada vez son más reiterados los estudios sobre la violencia escolar en el escenario nacional, dando cuenta de las distintas formas externalizadas en que este se construye en la sociedad cubana; y persisten enfoques conservadores para su atención como los enunciados con anterioridad. Si bien se han dado pasos de avances, con el posicionamiento del tema en los medios de difusión nacional, aún emergen brechas importantes en el orden de la política educativa para su comprensión.

Desde una mirada a la nueva Constitución de 2019 con el "tránsito de ese socialismo estadocéntrico hacia una visión multiactoral y de una organización descentralizada de la sociedad" (Espina, 2017, pp.3-4 en Rojas y García, 2017), los retos desde el trabajo preventivo aún persisten (Espina, 2014; Zabala, 2015a). En el sector escolar y las políticas sociales, estas emanan en las deficiencias para visibilizar la violencia a partir de los actores escolares y códigos que la configuran. Si bien es significativo el reconocimiento de la violencia intrafamiliar y la protección acentuada de la infancia, a tenor de los artículos 85, 86, 87 y 88 del capítulo III, y las garantías del inciso (a), artículo 94 e (i) del 95, ambas en el capítulo VI, los símbolos distintivos de la violencia escolar no se colocan en ellas y se continúan delimitando en función de influjos foráneos, lejos del espacio escolar, patentizando así las fisuras preventivas para su erradicación.

Otras incongruencias apuntan a la permanencia de disposiciones políticas contrapuestas con la realidad socioescolar contemporánea. Especialmente se alude a la "concentración por casi 8 horas en los colegios de los estudiantes, el carácter de trabajador social de los docentes, la inserción de las teleclases ${ }^{3}$, entre otras" (Ministerio de Educación, 2004, p.5). Las mismas, en su origen, hacia inicios del siglo XXI, aun cuando fueron pertinentes para promover una mayor atención al estudiante y enfrentar el déficit de maestro que experimentaba la sociedad, tienden a ser, en ocasiones, contraproducentes para la propia dinámica escolar contemporánea. Entre sus consecuencias imprevistas se advierte, indistinta de la derogación de "las teleclases" en la actualidad, la sobrecarga de la labor docente al potenciar cierta desvinculación de su rol principal, insuficiencias en la atención al estudiante, un deterioro del protagonismo del maestro en los centros, así como un distanciamiento en su vínculo con los colegiales.

De forma indirecta, las incongruencias enunciadas, también se acompañan de la desmotivación docente ante la falta de estímulos y reconocimientos de su labor. Asimismo, ellas, junto a la insatisfacción económica que experimentan los profesores (aun con el incremento salarial) han favorecido germinen prácticas negligentes en su accionar educativo. De esta forma,

\footnotetext{
${ }^{3}$ Término coloquial empleado para hacer alusión a las clases impartidas por los canales nacionales de la televisión cubana.
} 
el dejar hacer o "abandono pedagógico del estudiante", en ocasiones, desde su tránsito apresurado por el centro, más si se tipifica de problemático, se ha institucionalizado informalmente en la dinámica escolar. Lo expuesto, se reafirma desde el discurso de algunos docentes, quienes remiten: "Tú estás haciendo labor social de que este niño tiene problemas, de que este niño la conducta que tiene y no se hace na [...], y al final se graduó el niño con los mismos problemas" (entrevista en profundidad a profesora, tutora educativa, 34 años de experiencia). Además, alegan que "el profesor no tiene, como era antes, un lugar en la sociedad de respeto" (profesor, 40 años de experiencia).

Otros docentes, en cambio, refuerzan mecanismos represivos aprendidos y construidos en las interacciones con los colegiales, a partir de la vigilancia jerarquizada del infante, interconectada con formas de agresión física (golpes, cocotazos, empujones), verbal y psicológica (gritos, burlas, ofensas, humillaciones, discriminación, etc.); cual garantes de control y orden institucional. Elementos reflejados en el siguiente gráfico.

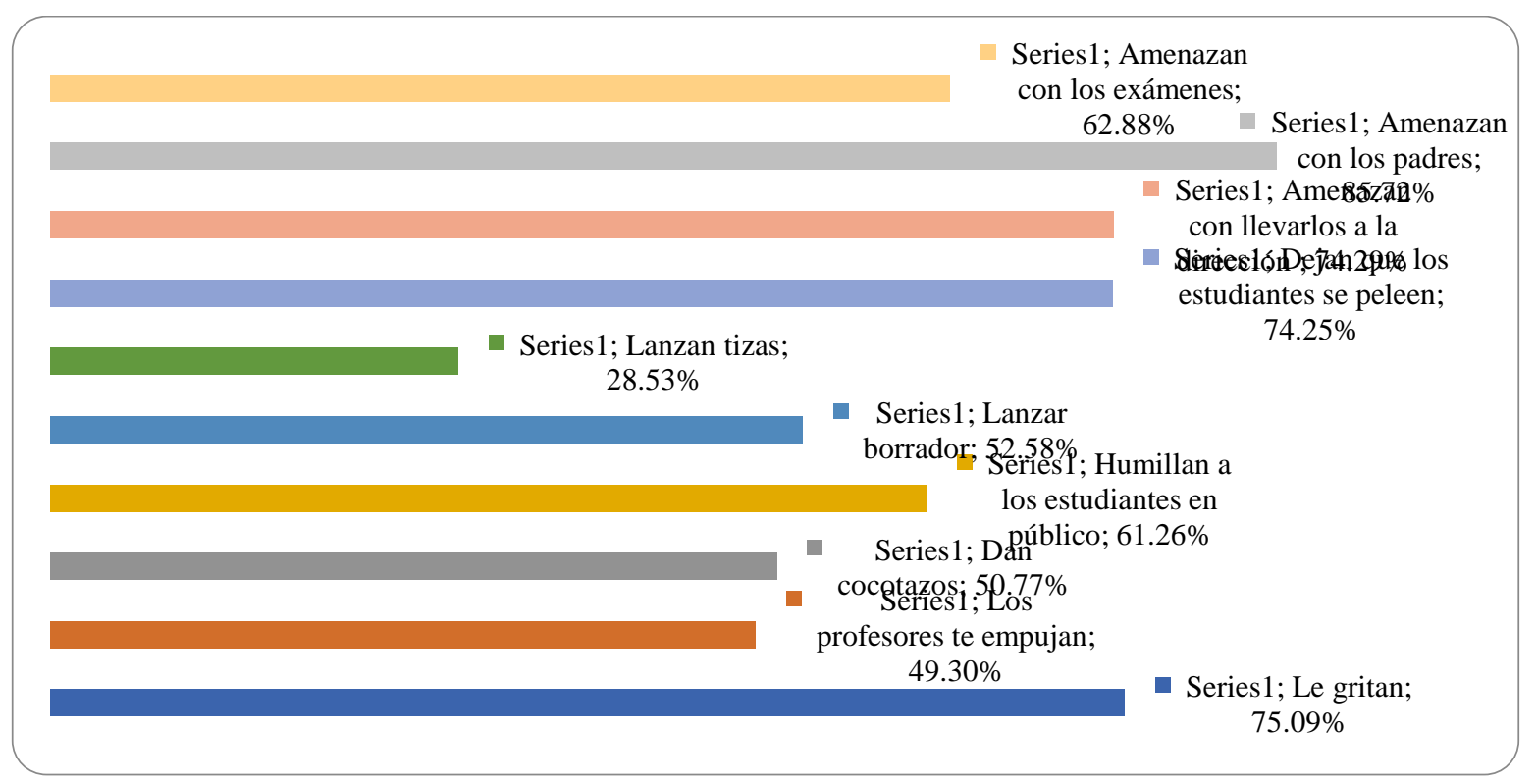

Figura 2: Gráfico 2- Amenazas, agresiones físicas y humillaciones que prevalecen hacia los estudiantes durante el proceso de enseñanza-aprendizaje. Elaborado por la autora a partir de los datos del cuestionario a estudiantes y profesores.

De forma general, el gráfico expuesto, da cuenta de las distintas tipologías de violencia que cobran vida en el contexto escolar durante el proceso de enseñanza- aprendizaje, y en que la figura del docente toma un lugar primordial. Sus manifestaciones, como parte de los mecanismos educativos implementados en sus interacciones con los colegiales, privilegian el uso de las amenazas a los estudiantes con sus padres, para un 85,72\%; los gritos, con un 75,09\%; amenazarlos con llevarlos a la dirección, con un 74, 29\%; dejar que los estudiantes se pelen y agredan, para un 74,25\%; amenazarlos con los exámenes con un $62,88 \%$, y humillar a los estudiantes en público. El resto de 
las prácticas puntualizadas, pese a su menor valor porcentual, recalcan las fisuras educativas que se deben limar. Retos importantes de nuestro sistema educación.

Lo significativo del ciclo constructivo de la violencia en el centro, ante la naturalización con que se perciben dichas prácticas deviene de la irrupción de intentos de agresión y amenaza de padres a profesores ante las prácticas implementadas hacia sus hijos. En corolario, se afirma por el 55\% de los docentes cómo delegan en estos toda la formación y preparación del escolar. Realidad que se revierte cuando se incumplen las normativas informales de socialización y educación articuladas durante el proceso de intercambio. Así, los padres “[...] cuando se da una situación con los muchachos, parten por la primera a quererle meter al profesor [...], y eso es lo mismo que hacen los hijos con los profesores" (entrevista a profesora, 58 años de experiencia). Ante dicha realidad, y como parte de las miradas sesgadas en torno a la violencia, los docentes no cuentan con un respaldo constitucional que vele por el resguardo y cuidado de su integridad.

En el marco del cuestionario a los familiares, cabe destacar, en contra parte, un cambio en las percepciones que sobre los docentes se tienen, las cuales se tiendan a fundamentar, además de lo expuesto por la institucionalización informal de las regalías docentes durante la jornada del educador. De esta manera, como muestra el grafico 3, y ante el incumplimiento sostenido de la Resolución Ministerial 506/14, sobre su prohibición, dígase las regalías; el trato diferencial a los escolares cual forma de discriminación, violencia psicológica y simbólica se coloca tangencialmente en el ordenamiento de la dinámica escolar.

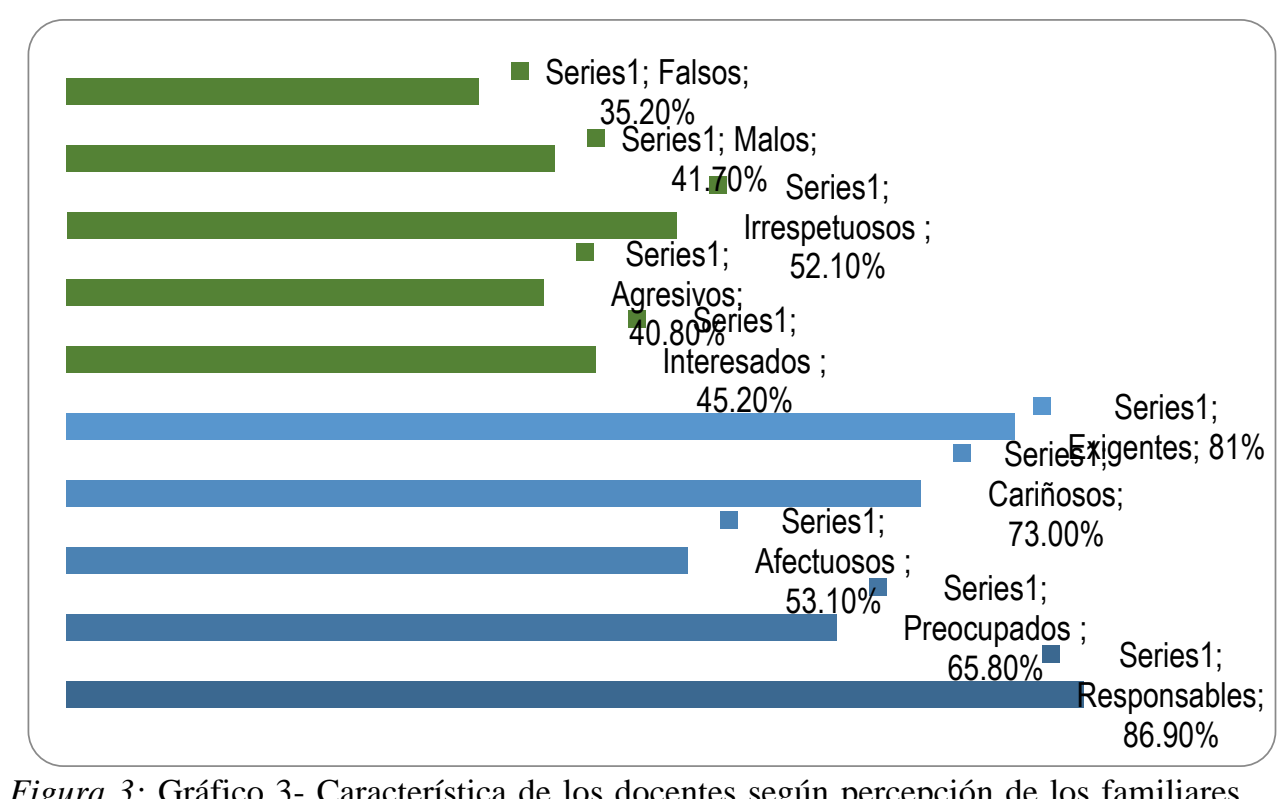

Elaborado por la autora a partir de los datos del cuestionario a familiares.

Los resultados de este gráfico, remiten transformaciones importantes en las percepciones familiares sobre los docentes e indirectamente sobre el contexto escolar. No obstante, cabe destacar que, la emergencia de nuevas características en algunos docentes, como el irrespeto (45\%), ser 
interesados (45.20\%), malos (41.70\%), agresivos (40.80\%) o falsos (35.20\%); aunque constituyen retos en la formación y preparación docente que se deben surcar, no logran desmeritar la imagen social que de su proceder prevalecen. Se alude a su responsabilidad (86.90\%), su exigencia (81\%), preocupación (65.80\%) y cariño (73\%) en el desempeño de su labor.

Por último, cabe destacar que, si bien uno de los logros de nuestra política educativa deviene de promover la participación de los sujetos en la construcción de su realidad escolar, la sectorización con que dicha participación se presenta y "su carácter alienante" (Rivero, 2013, p. 45) alerta de retos importantes que se han de surcar. Al respecto, cabría preguntar ¿cómo se concibe un proceso de participación del sujeto, en la construcción de su sistema escolar, si no participa en la conformación de las directrices políticas que la institucionalizan? o ¿en qué dirección y sector se propicia desde la política educativa la participación?

Una mirada a los resultados de los cuestionarios, pudiera remitir inicialmente un regocijo social, al destacarse por el $100 \%$ de los actores escolares su sentir cual actores constructores directos de su sistema de enseñanza; sin embargo, las demandas sociales por las que abogan y que implican un reordenamiento de su estructura organizacional, y de las estrategias de control del proceso docente, nos muestran brechas importantes en su proceder.

Entre los principales hallazgos se aborda, la necesidad de eliminar la existencia de una sola sección del proceso de enseñanza (de 7:30 am- 4.00 pm) donde se incluye la merienda escolar. Realidad en la práctica cotidiana, al decir de los entrevistados, que genera más trabajo para el docente, impidiéndoles, en ocasiones, cumplir su función social inicial. O sea, "mantener al estudiante recogido de la calle, a tenor de la desmotivación por el estudio presente en ellos como parte de las nuevas demandas y oportunidades sociales que, fuera del centro, existen" (profesora, 58 años de trabajo). A su vez, la precaria calidad en la formación de los profesores egresados, carentes de habilidades y motivación por el ejercicio del magisterio, tributa a la irrupción prácticas violentas hacia los colegiales como las descritas, pero independientemente de ello, su permanencia se hace necesaria consecuencia de la deserción docentes que aún persiste. Súmese, la pervivencia de la poca partición de la familia en los problemas que afectan a sus hijos, recayendo toda la atención del infante sobre el maestro y la escuela.

En resumen, como se refuerza en las investigaciones hacia la educación y los procesos de participación de los actores escolares, la violencia simbólica en el escenario educativo cubano se patentiza implícita en la comunicación vertical, autoritaria y asimétrica característica del proceso de enseñanza (Urguila, 2007; Castillo, 2008; Rivero, 2013). Elemento sustentado, en la dinámica válida de ser el Ministerio de Educación el que elabora las directivas, normativas y resoluciones pautadas por la política educativa nacional, al margen del diálogo con la ciudadanía (Rivero, 2013; 
MINED, 2004, 2015). No obstante, las lecturas críticas expuestas no demeritan la calidad de nuestro sistema educacional, al no constituir una generalidad, sino que advierten de fisuras imprevistas en su concreción y de las directrices requeridas para su atención.

\section{CONCLUSIONES}

La mirada crítica expuesta, alerta del reto a enfrentar en el reordenamiento de las políticas educativas, en función del contexto social en que las mismas se implementen. A su vez, dicho desafío implica reconocer fisuras significativas en sus disposiciones que, directa e indirectamente, favorecen a una reconfiguración de las relaciones escolares donde la violencia escolar toma lugar. Violencia expresa en el daño físico, verbal, psicológico y simbólico que emergen en los intercambios escolares de los escenarios santiagueros y que, en ocasiones, roza con prácticas fraudulentas y de corrupción en el centro. Al respeto, la presencia de la violencia en los centros, no solo se direcciona hacia las relaciones entre iguales como prevalece en la literatura, sino que, en su construcción, también intervienen docentes y familiares como actores activos del proceso. En consecuencia, con ello, los resultados obtenidos permiten contrastar la mirada relacional que prima en la comprensión de dicho flagelo, desde su visión integral entre el sistema normativo, las directrices de la política educativa, el sistema escolar, cognoscitivo, comunicativo y otros que en este convergen.

En otro orden de ideas, se hace necesario enriquecer las directrices de las políticas sociales en el ámbito educativo con las tendencias investigativas que en torno a la violencia escolar existen, en aras de hacer frente a sus diversas formas de emergencia y construcción. En tal sentido, urge elaborar o incorporar en los estatutos escolares (decreto y resoluciones) el posicionamiento categorial y conceptual de la violencia escolar, desde los diversos actores que en ella intervienen, junto a sus indicadores de evaluación y mecanismos de sanción para docentes y familiares que incurran en prácticas violentas en los centros escolares. Además de promover procesos de capacitación que reflejen la dimensión multidireccional en que dicha violencia se construye. El apoyo de los medios de difusión, junto a la voluntad política para atender esta temática, impone parafraseando a nuestro Comandante en Jefe Fidel Castro cambiar todo lo que debe ser cambiado; pero desde las realidades escolares donde el sujeto se construye, conforma la violencia y la dinámica escolar.

\section{REFERENCIAS BIBLIOGRÁFICAS}

Astor, R. (2017). Toda conducta de victimización necesita ser atendida, incluso, si es la primera vez: Entrevista a Ron Avi Astor. Centro de Investigaciones Avanzadas en Educación, Universidad de Chile, Chile.

Recuperado de: www.ciae.uchile.cl/index.php?page=view_noticias\&langSite=es\&id=1070 
Borroto, L. (2016). Cuba y las políticas públicas en educación. Revista Estudios del Desarrollo Social: Cuba y América Latina, 4(4), Número Extraordinario, 76-83.

Cala, C. (2019). La construcción social de la violencia escolar como relaciones recíprocas autoorganizadas (tesis de doctorado en Sociología). Centro de estudios cubanos y caribeños Dr. José Antonio Portuondo, Universidad de Oriente, Santiago de Cuba, Cuba.

Cala, C. y Benítez, D. (2018). Violencia interpersonal en al marco de las relaciones escolares. Visión sociológica desde los adolescentes santiagueros. Revista Santiago, Número Especial, 86-100.

Cala, C. y Caraballo, E. (2017). La construcción social de la violencia en jóvenes santiagueros. Revista Santiago, (144), 666-681.

Cala, C., Espronceda, M. y Betancourt, D. (marzo 2018a). Violencia escolar como condicionante de vulnerabilidad. Revista Atlante, 1-17 Recuperado de: www.eumed.net/rev/atlante/index.html

Cala, C., Espronceda, M. y Betancourt, D. (2018b). Violencia en ambientes escolares como expresión de vulnerabilidad (pp. 60-78). En Santana, Y. y Martínez, A. (2018), Vulnerabilidad e Inclusión social: Miradas encontradas. España: Editorial Academia Española.

Campañat, E. (2017). Maltrato Infantil entre pares (Bullying). Conferencia impartida en el Policlínico Camilo Torres Restrepo, Santiago de Cuba, Cuba.

Castillo, S. (2008). Las potencialidades transformadoras de la concepción pedagógica estratégica "los chicos del barrio", en la atención educativa a menores en institución de desventaja social (tesis de doctorado en Ciencias Pedagógicas). Instituto Central de Ciencias pedagógicas (ICCP), La Habana, Cuba.

Chávez, J. y Deler, G. (2013). Antología del pensamiento educacional de la revolución cubana. La Habana, Cuba: Sello Editor Educación Cubana.

Constitución de la República de Cuba 1976. Gaceta Oficial de la República de Cuba, versión digital. Recuperado de: www.gacetaoficial.gob.cu/html/constitucion_de_la_republica.html

Constitución de la República de Cuba (2019). En Gaceta Oficial de la República de Cuba, versión digital. Recuperado de: www.granma.cu/file/pdf/gaceta/Nueva\%20Constitución\%20240\%20KB-1.pdf

Cordoví, Y. (julio -diciembre 2009). La disciplina escolar desde el pensamiento pedagógico en Cuba, 1793-1898: consideraciones metodológicas. Projeto História, São Paulo, (39), 289-293.

Recuperado de: www.revistas.pucsp.br $>$ revph $>$ article $>$ download

Cordoví, Y. (2012). Cuerpo, pedagogía y disciplina escolar en Cuba. Dispositivo de control desde los discursos higienistas (1899-1958). Tzinzun, (57),86-103.

Denzin, N. (1970). Sociological Methods: a Source Book. Chicago: Aldine Publishing Company.

Decreto Ley no. 64 (1982). Del sistema para la atención a menores con trastornos de conducta. Ministerio de Educación, La Habana, Cuba

Espina, M. (2014). Desigualdad social y retos para una nueva institucionalidad democrática en la Cuba actual. Espacio Laical, Número especial, 101-104. Recuperado de: espaciolaical.org/contens/38/101104.pdf

Fabregat, M. (2016). El sistema de educación en cuba: raíces, logros y retos en el siglo XXI. Revista Nuances: estudos sobre Educação, Presidente Prudente-SP, 27(1),187-202.

Garcés, R. (2015). Homofobia: diagnóstico preliminar de un tema pendiente. Sexología y Sociedad, 21(1), $31-48$.

García, G. J. (1998). Bosquejo histórico de la educación en Cuba. La Habana, Cuba: Editorial de Libros para la Educación.

González, P. y Reyes, R. (2010). Desarrollo de la Educación en Cuba después del año 1959. Revista Complutense de Educación, 21(1), pp.13-35.

Guevara, A., Ferrer, D., Norman, L. y Suz, M. (2016). Adolescentes, violencia y relaciones interpersonales. Un análisis en estudiantes de nivel medio de la ciudad de Santa Clara. II Congreso Internacional de Investigadores sobre Juventud. Palacio de Convenciones de la Habana Cuba. 
Hernández, R. (2014). Metodología de la investigación. 6ta ed. S.A. de C.V. México: McGraw-Hill / Interamericana Editores.

Labacena, Y. (2015): Padre y abuelo a la vez. Periódico Juventud Rebelde. Recuperado de: www.juventudrebelde.cu/cuba/2015-07-02/padre-y-abuelo-a-la-vez

Labacena, Y. y Tamayo, L. (2018). Reordenan trabajo por cuenta propia en Cuba. Periódico Juventud Rebelde. Recuperado de: www.juventudrebelde.cu/media/cover-de-noticia-

Lineamientos de la Política Económica y Social del Partido y la Revolución para el período 2016-2021, julio 2017. Recuperado de: www.granma.cu > pdf > gaceta > Lineamientos 2016-2021 Versión Final

Ministerio de Educación (MINED) (2004). El desarrollo de la Educación. Informe Nacional. Ministerio Nacional de Educación. Recuperado de: www.oei.es/historico/pdf2/informe-cuba-ibe.pdf

Ministerio de Educación Superior (2015). Revisión Nacional 2015 de la Educación para todos: Cuba. La Habana, Cuba. Recuperado de: www.acaoeducativa.org.br/desenvolvimento/wpcontent/.../11/Informe_Cuba.pdf

Naranjo, Y., Figueroa M. y Cañizares, R. (2015). Envejecimiento poblacional en Cuba. Gaceta Médica Espirituana, 17 (3), 223-233. Recuperado de: revgmespirituana.sld.cu/index.php/gme/article/view/830/html_79

Oficina Nacional de Estadística e Información [ONEI] (2019). Educación: Resumen del curso escolar 2017/ 2018 e inicios del curso escolar 2018/2019. La Habana, Cuba: Edición junio 2019.

Olweus, D. (2011, 3 de mayo). Bullying at school and later criminality: Findings from three Swedish community samples of males. Criminal Behavior and Mental Health, 151-156. Recuperado de: doi.org/10.1002/cbm.806

Pérez, G. (2017). Manifestaciones y factores de la violencia en el escenario escolar TELOS. Revista de Estudios Interdisciplinarios en Ciencias Sociales, Universidad Privada Dr. Rafael Belloso Chacín, 19 (2), 237-259.

Regueira, K. (2008). Estrategia educativa para la prevención de la violencia escolar en la formación inicial intensiva del profesor general integral de secundaria básica (tesis de doctorado en Pedagogía), Departamento de Ciencias de la Educción, Universidad de Ciencias Pedagógica "Frank País García", Santiago de Cuba, Cuba.

Resolución Ministerial no.139 (2011). Educación sexual. Ministerio de Educación, La Habana, Cuba. Recuperado de: www.mined.gob.cu $>$ document $>$ rm-139-del-2011-educacion-sexual

Resolución Ministerial no.11 (2012). Legislación de los reglamentos escolares. Ministerio Nacional de Educación, La Habana, Cuba. Recuperado de: juriscuba.com > organismos-estatales-2 > educacion

Resolución Ministerial no.111 (2017). Del trabajo preventivo. Ministerio de Educación, La Habana, Cuba. Recuperado de: www.mes.gob.cu > resoluciones

Rivero, Y. (2013). Participación educativa en las escuelas primarias de la Cuba actual (tesis de doctorado en Sociología). Departamento de Sociología de la Universidad de La Habana, La Habana, Cuba.

Rojas, F. y García, C. (2017). Entrevista a Mayra Espina: Por un socialismo donde el conocimiento se vuelva política. Revista Temas, cultura ideología y Sociedad. Recuperado de: www.temas.cult.cu/catalejo/mayra-espina-por-un-socialismo-donde-el-conocimiento-se-vuelvapol-tica

Rodney, Y. (2010a). Estrategia pedagógica dirigida al profesorado para la prevención de la violencia escolar (tesis doctoral en Pedagogía) Universidad de Ciencias Pedagógicas “Enrique José Varona”, La Habana, Cuba.

Rodney, Y. (2010b). La preparación del profesorado en la prevención de la violencia de género desde la escuela. Ponencia presentada en el 5to congreso Internacional de Educación, orientación y terapia sexual. La Habana, Cuba.

Rodney, Y. y García, M. (2014). Estudio histórico de la violencia escolar. Revista VARONA, (59), 41-49. 
Rodney, Y. y García, M. (2015). Políticas públicas sobre violencia escolar en Cuba: entre lo jurídico y la realidad. Sexología y Sociedad, 21(1), 146-180.

Rodney, Y., Lorenzo, K., Cruz, Y. y Muñoz, J. (2015). Educar para la Igualdad. Propuesta metodológica para la prevención y atención educativa de la violencia de Género en el contexto escolar. La Habana, Cuba: Uniceff, UNESCO.

Rodríguez, L. y Ramos, A. (2018). Intervención educativa a profesores de Secundarias Básicas sobre el Manejo del Bullying entre estudiantes. Ponencia del III Congreso Internacional de Estudios sobre la Juventud. Palacios de las Convenciones, La Habana, Cuba.

Ruiz, J. (1999). Metodología de la investigación cualitativa. España: Universidad de Deusto, Bilbao

Torres, N.; Arteaga, S.; Moya, Y. et al (2019). Estrategia educativa para la formación de una cultura de paz. Ponencia I Convención Internacional Ciencia y Conciencia 2019, Universidad de Oriente, Santiago de Cuba, Cuba.

Santiesteban, M. (2013). La gestión educativa de prevención de la violencia universitaria en la formación inicial del profesional de la educación (tesis doctoral en Ciencias de la Educación). Santiago de Cuba, Universidad de Ciencias Pedagógicas "Frank País García", Cuba.

Sampedro, P. (2017). Caracterización de la vivencia sobre la violencia de un grupo de adolescentes capitalinos. Ponencia presentada en el Simposio Nacional del centro de estudios de Investigaciones Psicológicas y Sociológicas de Cuba. Palacio de Convenciones de La Habana, Cuba.

UNESCO. (2019). La violencia y el acoso escolares son un problema mundial. Recuperado de: https://es.unesco.org/.../violencia-y-acoso-escolares-son-problema-mundial-segun-nue..

Urguila, M. (2007). Resultado integral del escolar. Estudios de algunas variables que influyen en el mismo (tesis de grado). Facultad de psicología, Universidad de La Habana, Cuba.

Vargas, A. (1999). Notas sobre el Estado y las políticas públicas. Bogotá: Almudena.

Velásquez, R. (2009). Hacia una nueva definición del concepto "política pública". Desafíos (20).

Zabala, M. (2008). Análisis de la dimensión racial en los procesos de reproducción de la pobreza. El rol de las políticas sociales para favorecer la equidad social en Cuba. Revista Pobreza, exclusión social_final, 397-422. Recuperado bibliotecavirtual.clacso.org.ar/ar/libros/clacso/crop/zabala/20zaba.pdf

Zabala, M. (2015). Equidad social y cambios económicos en Cuba: retos para la atención a la pobreza y las desigualdades. En Espina, M. \& Echevarría, D. (Coords.). Cuba: los correlatos socioculturales del cambio económico. La Habana: Ruth Casa Editorial.

Zabala, M., Fundora, G., Camejo, D., Díaz, D. y Hidalgo, V. (2018). Referentes teóricos para el estudio de las desigualdades sociales en Cuba: reflexiones sobre su pertinencia. Revista Estudios del Desarrollo Social: Cuba y América Latina, 6(1), 86-118.

\section{Agradecimientos / Acknowledgments:}

Expreso mi agradecimiento a los docentes del Departamento de Sociología de la Facultad de Ciencias Sociales a nivel de licenciatura y posgrado.

Conflicto de intereses / Competing interests:

La autora declara que no incurre en conflictos de intereses.

Rol de los autores / Authors Roles:

No aplica.

Fuentes de financiamiento / Funding:

La autora declara que no recibió un fondo específico para esta investigación.

Aspectos éticos / legales; Ethics / legals:

La autora declara no haber incurrido en aspectos antiéticos, ni haber omitido aspectos legales en la realización de la investigación. 